\title{
Recent Advances in Carbon Nanotubes for Nervous Tissue Regeneration
}

\author{
Carlos Redondo-Gómez $\mathbb{D}^{1},{ }^{1}$ Rocío Leandro-Mora, ${ }^{2}$ Daniela Blanch-Bermúdez, ${ }^{2}$ \\ Christopher Espinoza-Araya, ${ }^{2}$ David Hidalgo-Barrantes, ${ }^{2}$ and José Vega-Baudrit $\mathbb{D}^{1,2}$ \\ ${ }^{1}$ National Laboratory of Nanotechnology LANOTEC, 1174-1200 Pavas, San José, Costa Rica \\ ${ }^{2}$ Faculty of Exact and Natural Sciences, National University of Costa Rica (UNA), Heredia, Costa Rica \\ Correspondence should be addressed to José Vega-Baudrit; jvegab@gmail.com
}

Received 10 October 2019; Revised 5 December 2019; Accepted 19 December 2019; Published 11 February 2020

Guest Editor: Ernesto Di Maio

Copyright (C) 2020 Carlos Redondo-Gómez et al. This is an open access article distributed under the Creative Commons Attribution License, which permits unrestricted use, distribution, and reproduction in any medium, provided the original work is properly cited.

\begin{abstract}
Regenerative medicine has taken advantage of several nanomaterials for reparation of diseased or damaged tissues in the nervous system involved in memory, cognition, and movement. Electrical, thermal, mechanical, and biocompatibility aspects of carbonbased nanomaterials (nanotubes, graphene, fullerenes, and their derivatives) make them suitable candidates to drive nerve tissue repair and stimulation. This review article focuses on key recent advances on the use of carbon nanotube- (CNT-) based technologies on nerve tissue engineering, outlining how neurons interact with CNT interfaces for promoting neuronal differentiation, growth and network reconstruction. CNTs still represent strong candidates for use in therapies of neurodegenerative pathologies and spinal cord injuries.
\end{abstract}

\section{Introduction}

The emergent field of nanomedicine proposes the application of precisely engineered nanomaterials for the prevention, diagnosis, and therapy of certain diseases, including neurological pathologies [1]. These pathologies occur when basic units of the nervous system start to deteriorate. In these nerve cells, alterations cause them to function abnormally, which results in demise of cell functions. Initial symptoms of neuronal deterioration may include loss of coordination or the ability to remember names, which may worsen over time if a large number of neurons deteriorate [2]. Due to the complexity of the nervous system, recovering function of the injured nerves or repairing damages associated to neurodegenerative conditions is still a major challenge in the biomedical field. Neurodegenerative diseases affect over 90,000 people every year, from which spinal cord injuries alone affect 10,000 people yearly. Alzheimer's and Parkinson's disease are the most common neurological diseases and occur in more than 5 million and 1.2 million Americans, respectively [3]. Considering the high amount of nerve repair procedures being currently conducted, as well as an increasing and ageing world population, the number of patients in need of neural implants to improve the regeneration of damaged tissue will only substantially increase over the years.

Neuroregeneration is the regrowth, restoration, or repair of degenerated nerves and nervous tissues, associated with the production of new axons, neurons, glia, myelin, and synapses. The nervous system is divided into the peripheral nervous system (PNS), which has the innate capability for self-repair and regeneration, while the central nervous system (CNS) is unable to self-repair and regenerate. Siliconbased materials are the most common for peripheral nerve implants; being studied since the 1960s, they have been used as a model system giving fundamental insight on nerve tissue regeneration.

Silicone has been implemented in the diagnosis, monitoring, and continuous treatment of nerve tissue damages [4]. Silicone been primarily used due to its biocompatibility, flexibility, and wide availability in different dimensions. However, their low impermeability and general inert 
properties do not actively prompt neural tissue regeneration and have led to research on substitute materials [5]. Today, the treatment for damages in the CNS (i.e., spinal cord) consists on physical therapy to help patients with limited mobility without a full regain and restoration of the tissue and motor function [6].

Although still narrow, the application of nanotechnology and nanomaterials to neuroscience has experienced an impressive growth over the past decades, with an increasing amount of studies proposing scaffolds based on nanomaterial as strategies to regenerate nerve cells and tissues [7]. An ideal scaffold for neural tissue application shall exhibit electrical activity to stimulate cell outgrowth, biodegradability, and bioactivity for growth factor delivery; interestingly, a number of nanomaterials exhibit some of these properties and have proved relative long-term success when implanted [8].

Carbon-based nanomaterials (CBNs) have shown great potential when interacting with neurons and nerve tissues [9-11]. The discovery and manipulation of innovative nanomaterials, like fullerenes and graphene, but especially carbon nanotubes (CNTs), are likely to have a major impact on neuroregenerative techniques and in the biomedical applications in general. CNTs have shown to interact with the nervous system promoting the neural development. Furthermore, the outstanding mechanical, thermal, and conductive properties make CNTs very promising for other technological fields as conductive composites and sensors [12]. This work intends to review some recent uses of CNTs in nerve regeneration. We start providing an overview of nerve architectures and recent progresses on carbon-based nanomaterials for regenerative therapies and neuron repair. We finish the review with some closing remarks on biocompatibility and toxicity challenges of CNTs when used as part of these therapies.

\section{Central and Peripheral Nerve Regeneration}

The human nervous system consists of the central nervous system (CNS) and the peripheral nervous system (PNS), which at the same time is composed of two cell types, neurons and neuroglia. Neurons are the brain's nerve cells that transmit information from electrical and chemical signals throughout the nervous system, while neuroglia are the most numerous cells and their purpose is to aid the function of neurons. Within these cells, there are Schwann cells in the PNS and astrocytes and oligodendrocytes in the CNS. Researchers have shown that functional and structural recovery of the nerves depends on both extrinsic and intrinsic factors [13].

2.1. Peripheral Nervous System Repair. The PNS consists of a complex collection of spinal nerves, brain nerves, and neuron clusters called ganglia. These cells interact with other tissues transmitting sensory messages to and from the spinal cord [14]. The PNS has the ability to slowly regenerate on its own (axon growth $0.5-1 \mathrm{~mm} /$ day); in case of small injuries, nerve axons can regenerate by proliferating Schwann cells and macrophages to remove cellular debris from the injury side. Schwann cells infiltrate in the injury to stimulate and guide the new forming axon across the damaged nerve [15]. On the other hand, larger injuries need to be surgically treated, commonly using an autologous nerve implant. While extrinsic factors contemplate the environment at the injury site, intrinsic factors like the size of the injury and the ability of the neurons to regenerate by the synthesis of growth factors influence PNS regeneration [14-16]. In general, tissue engineering together with nanotechnology, aim to create innovative materials to help accelerate the PNS recovery since the delay in tissue regrowth may lead to muscle atrophy.

2.2. Central Nervous System Repair. The CNS includes the brain and the spinal cord, it is responsible of interpreting and conducting signals as well as providing stimulation to the PNS and from there to other tissues [17]. In contrast to the PNS, the CNS does not support full tissue regeneration; this leads to permanent loss of functions that can cause several physical and cognitive complications. Many factors such as the environment surrounding the CNS injuries and lack of neuronal regeneration capacity prevent cells from regenerating. Axonal regrowth is constrained by supporting cells, like myelinating oligodendrocytes, that create a growth inhibitor environment due to the formation of glial scar tissue and the lack of Schwann cells to promote axonal growth [18]. Therefore, the overall regeneration strategies for CNS are to reactivate gliosis while promoting tissue regeneration, where nanomaterials incorporated as part of current implants may help with [6].

\section{Current Materials for Nerve Tissue Regeneration and Stimulation}

Neurological implants' success in enhancing survival of damaged neurons, axons growth, and neuronal synaptic signal transmission is key to face the functional impairment that caused neuronal loss or degeneration. Basically, any strategy developed to fix an injury on the CNS should focus on regrowing injured axons, the plastic remodeling of neuronal circuitry, and the construction of new neurons [7].

Upgrades in material synthesis have allowed to develop artificial nerve conduits built of absorbable synthetic materials. Materials like polyhydroxybutyrate (PHB), polylactic acid (PLA), or polyglycolic acid (PGA) are being investigated as biodegradable-absorbable synthetic polymers for neural cell growth and axon organization. In fact, absorbable synthetics (PLA and PGA), and nonabsorbable synthetics like poly lactic-co-glycolic acid (PLGA), are already used for nerve regeneration. These three polymers are mechanically fragile and lack regions suitable for further chemical modification [19]; in any case, they have been FDA-approved for use in several neuron repair devices.

3.1. Polycaprolactone- (PCL-) Based Materials. PCL has gained considerable interest in the field of nerve regeneration research. The main features of this biodegradable 
polyester are its ease of manipulation and low processing costs. Its high processability is given by the great solubility this substance has in many organic solvents and that its crystalline nature enables easy formability at low temperatures. Neurolac ${ }^{\circledR}$ (Polyganics Inc., the Netherlands) is a PCL nerve conduit approved by the FDA [20].

Other PCL co-polymers like the biodegradable polycaprolactone fumarate (PCLF) have recently allowed the fabrication of CNT composites [21]. These PCLF-CNT scaffolds not only exhibited excellent suitability to culture neuroblastic PC-12 cells (that can easily differentiate into neuron-like cells), but also allowed for good cell growth, differentiation, and electrical stimulation, which reflected in a neurite extension and promoted cellular migration and intracellular connections, which are all critical cellular behaviours for nerve regeneration [21].

3.2. Collagen-Based Materials. Collagen comprises a large family of proteins with a wide range of biomedical uses including peripheral nerve repair. When the purified collagen becomes weakly antigenic, it exhibits a smooth microgeometry and transmural permeability, both support the diffusion processes through collagen matrices [22]. Collagen type I constitutes an essential structural component of the extracellular matrix and has been employed at fabricating nerve repair conduits. Of over a dozen nerve conduits, currently, FDA-approved three are made of collagen type I: NeuraGen, NeuroMatrix, and NeuroFlex [22].

The first semipermeable type I collagen nerve guidance conduit approved by the FDA was NeuraGen ${ }^{\circledR}$ (Integra Life Sciences Corporation, Plainsboro, NJ, USA). A medical study on peripheral nerve reconstruction reported the clinical experience of using this implant, in which patients tolerated splinting and exercise without negative clinical repercussions. In another research, this conduit was compared with direct suture repair, in patients with complete traumatic nerve injuries. Results showed that patients who were treated with NeuraGen ${ }^{\circledR}$ had less postoperative pain than those treated with direct suture repair. The main conclusion was that nerve repair using the NeuraGen ${ }^{\circledR}$ is a quite effective method of joining severed nerves [23]. As collagen-based nerve repair conduits still lack good mechanical stability, the possibility to reinforce them with CNTs seems appealing; in fact, this might improve their electrical conductivity, thus exhibiting good viability of neuronal cells as has been demonstrated in similar biomaterials [24].

\section{Carbon Nanomaterials for Nerve Tissue Regeneration}

Nanosized materials and nanoscale technologies seem to challenge many traditional paradigms in Materials Science. Since the discovery of CNTs by Sumio lijima in 1991, scientific literature on the physical and chemical properties of nanomaterials, especially carbon-based nanomaterials (CBNs), has grown significantly and so has the use of CBNs in nerve regeneration applications (Figure 1).
CBNs offer unequal advantages, like high electrical conductivity, high surface-volume ratio, powerful mechanical strength, and chemical stability [25]. CBNs are held in high esteem in the biomedical materials community, and constant efforts have been made to integrate them into existing materials and devices like cellular sensors, tissue scaffold reinforcements, and drug delivery systems [26]. Fullerenes, graphene and carbon nanotubes (CNTs) are the most studied CBNs; they have attracted significant attention regarding their unique optical, electronic, mechanical, thermal, and chemical properties [27].

4.1. Fullerenes. Fullerenes are CBNs of great importance in biomedical research. Since their discovery in 1985, it was evident that this polyaromatic, symmetrical, and hollow spherical cage $\mathrm{C}_{60}$ molecule was meant to find a number of versatile applications in antiviral therapies [28], energy production, flat panel displays, semiconductors, environmental technologies, cosmetics [29], and food industry [30]. $\mathrm{C}_{60}$ has been recently used as an in vitro vehicle for therapeutic astrocyte delivery to neural lesions [31]. PCL and $\mathrm{C}_{60}$ were electrospun into $200 \mathrm{~nm}$ diameter nanofibers that showed good cell attachment and promising potential as drug delivery devices (Figure 2) [31].

4.2. Graphene. Graphene is a polycyclic aromatic molecule that is composed of a two-dimensional sheet of $\mathrm{sp}^{2}$-bonded carbon atoms. This dimensional feature grants graphene and its derivative graphene oxide (GO) with high elasticity, conductivity, remarkable mechanical strength, rapid heterogeneous electron transfer, and high surface area [26]. Graphene is considered a versatile building block for functional nanoelectronics, energy storage, and production [33] as well as antibacterial [34], biosensing [35], and anticancer therapies [11].

Different amounts of layers grant the graphene with different properties, going from one-layered graphene to multilayered graphene structures [36], and interaction between these graphene-based nanomaterials and neurons have been recently explored [35]. In a recent study by Pampaloni and coworkers, it is shown that single-layer graphene (SGL) can tune astrocytes excitability and increases neuronal firing by altering membrane-associated functions in vitro. The authors hypothesize that graphene restricts the mobility of $\mathrm{K}^{+}$ions in close proximity to the SGL surface, but only when SLG is deposited on electrically insulating substrates. In this fashion, graphene properties might affect neuronal information processing (Figure 3) [35].

4.3. Carbon Nanotubes (CNTs). Carbon nanotubes (CNTs) are most widely used in nerve regeneration $\mathrm{CBN}$ to date [22]. Different from other CBNs, CNTs exhibit tunable physical (length, diameter, single-walled SWCNTs, vs multiwalled MWCNTs, chirality) [38] and chemical properties (surface functionalization and high electrochemical surface area) [26]. CNTs can be envisioned as cylinders made of rolled-up 


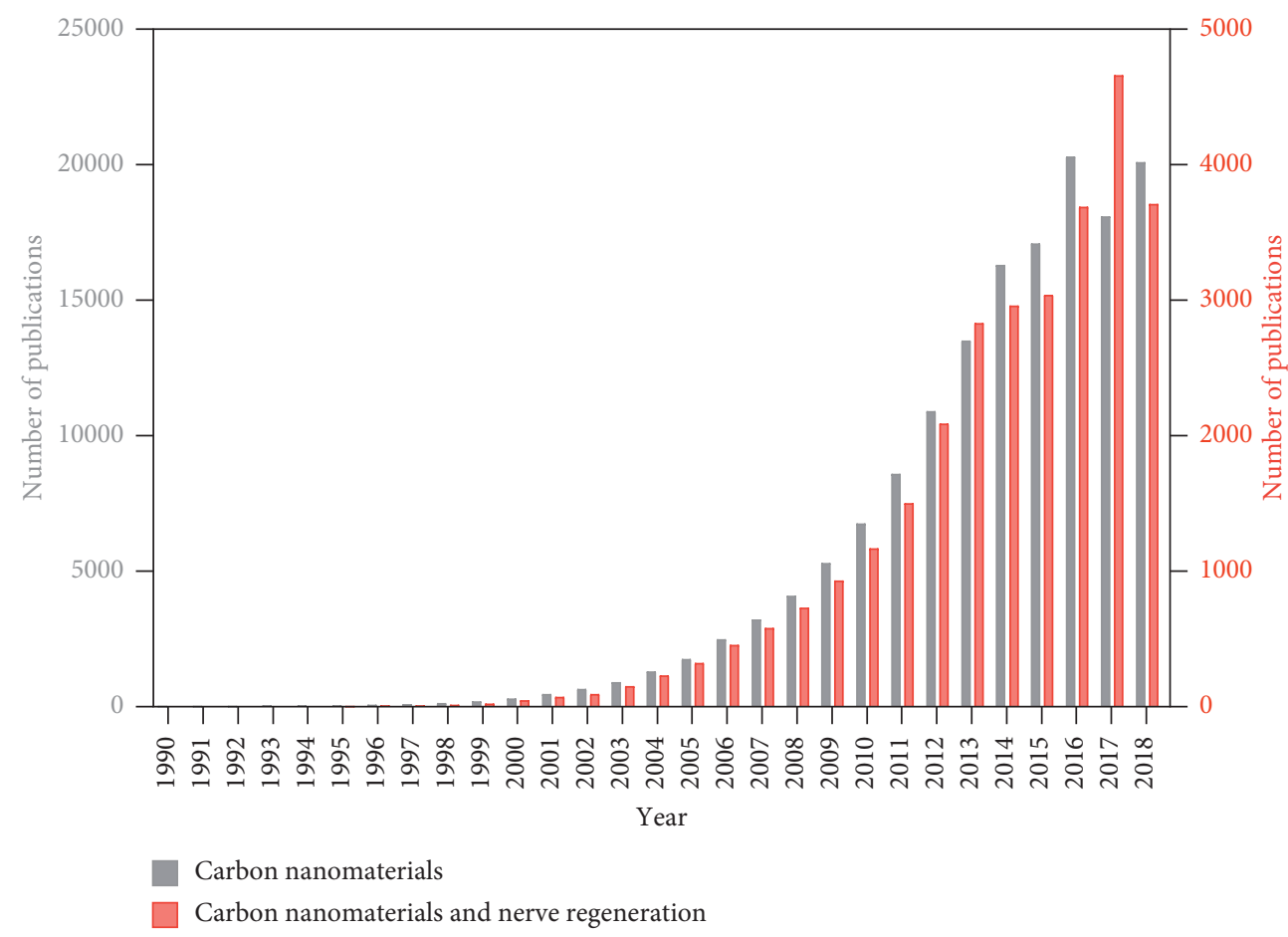

FIGURE 1: Increasing trend of reports on carbon nanomaterials (grey) and carbon nanomaterials and nerve regeneration (red). A google scholar search was performed on either "carbon nanomaterials" or "carbon nanomaterials and nerve regeneration" on the specified time period.

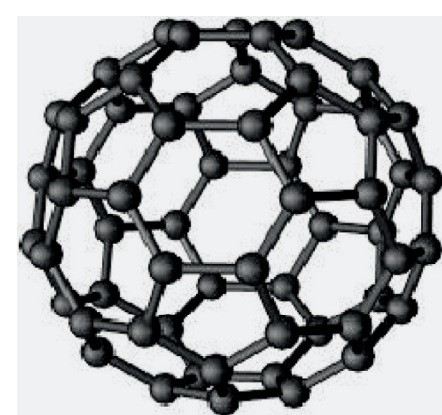

(a)

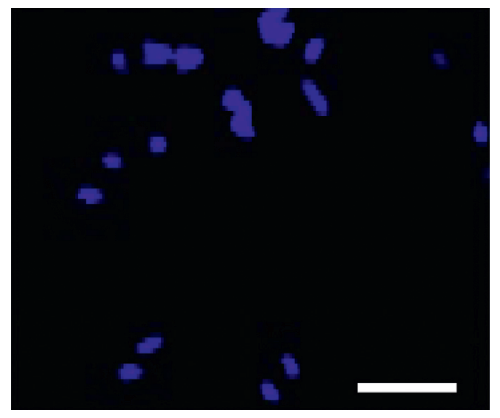

(d)

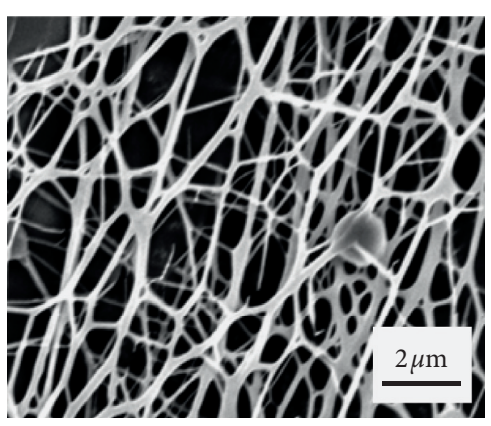

(b)

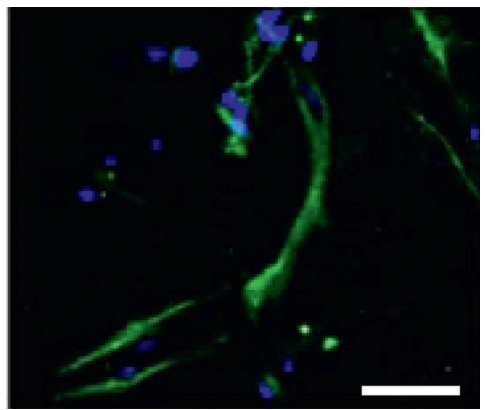

(e)

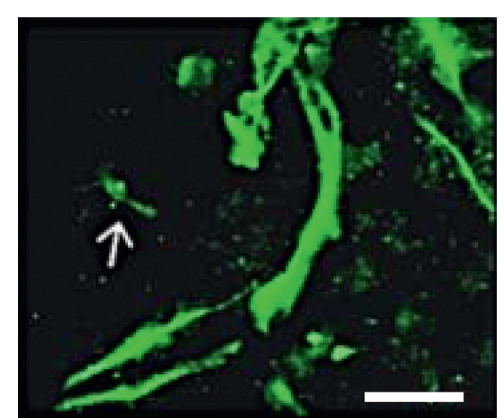

(c)

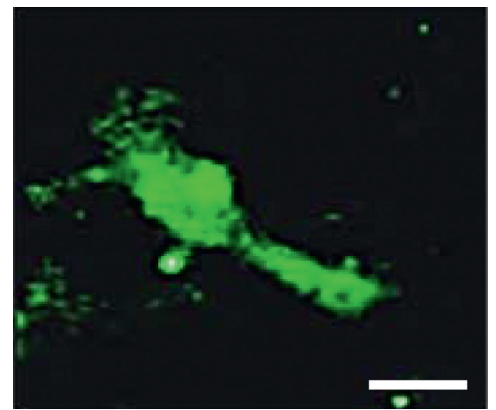

(f)

Figure 2: (a) Representation of $\mathrm{C}_{60}$ fullerene [32]. (b) SEM images of PCL-fullerene nanoscaffolds fabricated by electrospinning. (c-e) Immunocytochemical micrographs of astrocytes cultures on the scaffolds and labeled with antiglial fibrillary acidic protein antibody (antiGFAP, green) and Hoechst dyes (blue, scale bar: $50 \mu \mathrm{m}$ ). (f) Magnification images of cells pointed with an arrow in (c) (scale bar: $25 \mu \mathrm{m}$ ) [31]. 


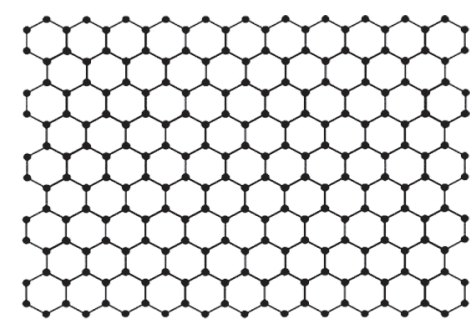

(a)

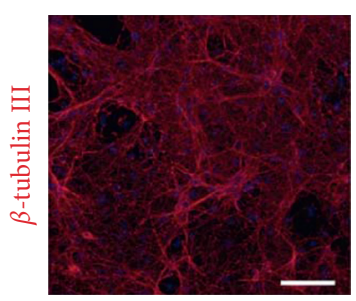

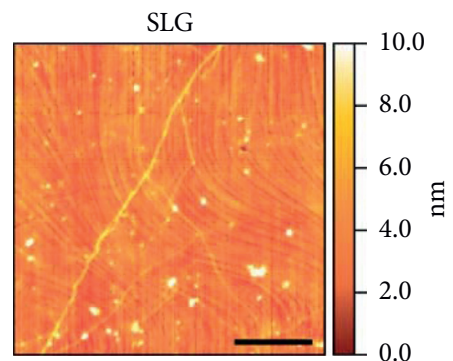

(b)

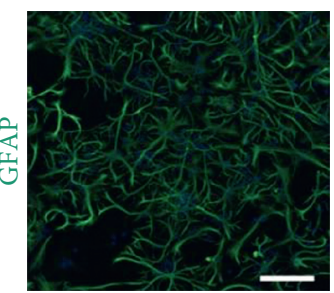

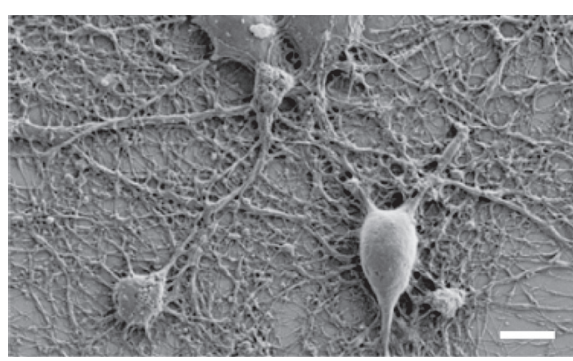

(c)

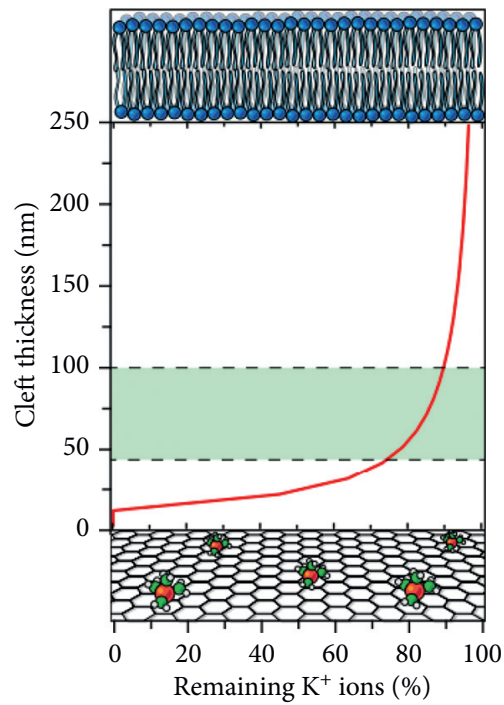

(e)

Figure 3: (a) Molecular representation of graphene [37]. (b) AFM topography of single-layer graphene (SGL, scale bar: $5 \mu \mathrm{m}$ ). (c) Scanning electron micrograph depicting hippocampal neuron morphology cultured onto SLG (scale bar: $10 \mu \mathrm{m}$ ). (d) Fluorescent microscopy images showing dissociated hippocampal networks labelled with class III $\beta$-tubulin (for neurons) in red and GFAP (for astrocytes) in green (scale bar: $100 \mu \mathrm{m}$ ). (e) Sketch of the local amount of $\mathrm{K}+$ depletion in the space between the cell membrane and the SLG surface (membrane/surface cleft) due to graphene trapping as function of cleft thickness. The light green region shows the extrapolated $\mathrm{K}+$ depletion values (red line) within the range of the estimated cleft dimensions (40-100 nm). See Reference [35] for more details.

graphene layers with diameters in the nanometer scale (Figure 4). CNTs possess an extended conjugated $\mathrm{sp}^{2}$ carbon network that renders a $\pi$-electron system which extends over the nanostructure originating either highly conducting CNTs or semiconducting ones; this also provides tunable band gaps compatible with neural activity [9]. The following section addresses the specific use of CNTs in neural regeneration and stimulation in deeper detail.

\section{CNTs for Neural Regeneration and Stimulation}

CNTs are generating an attractive approach in the treatment of neural pathologies and nerve tissue damaged. On top of their aforementioned capabilities, CNTs show morphological similarity to neurites, and small CNT bundles have dimensions similar to those of dendrites (the branched extensions of neuron cells), enhancing possibilities for not only probing, repairing, stimulating, or reconfiguring neural networks [9] but also gaining insights into basic mechanisms of neuronal functions [10]. Success on the application of
CTNs is strongly connected to the ability to control the interaction between them and the neurons' changes in ionic conductance and synaptic transmission, this being a perk when incorporated into electrodes and conductive probes $[1,40]$. In the following sections, we provide a comprehensive view of the use of CNTs in neural regeneration and stimulation.

5.1. Improving CNTs Neurocompatibility. CNTs are synthesized through a number of treatments that renders them positive or negative charged and be further modified to incorporate various functional groups via covalent [41] and noncovalent pathways [42].

Incorporation of hydrophilic polymers such as polyethylene glycol (PEG) increases CNT solubility in aqueous solution, and increasing their biocompatibility and facilitating the fabrication of CNT-based medical materials and common use polymers such as poly-ethyleneimine (PEI) and poly-L-ornithine (PLO) have been reported to promote neural attachment and subsequent neurite outgrowth; these noncovalent functionalization examples represent a valuable 


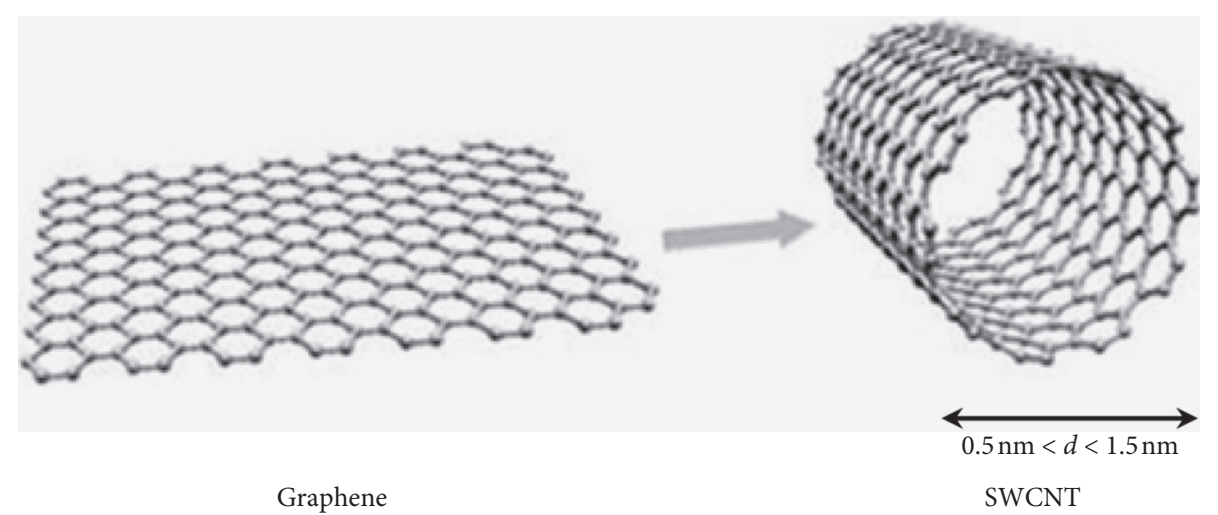

(a)

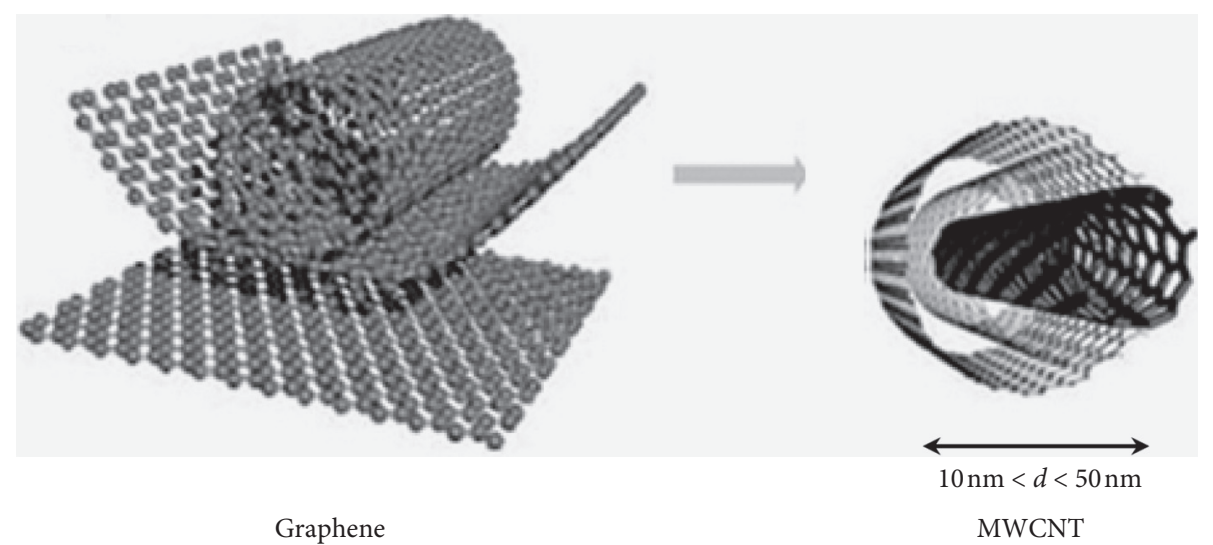

(b)

FIGURE 4: Representation of a typical (A) single-walled carbon nanotube (SWCNT) and a multiwalled carbon nanotube (MWCNT) (adapted from reference [39]).

resource when manufacturing the coating for neural interface devices $[43,44]$. Use of CNTs decorated with these poly-cations generally promotes neuron growth, and this is most likely due to an enhanced electrostatic interaction between the CNTs and the plasma membrane of neural cells that has a negative charge [45]. Recent biofunctionalization approaches [46] appear as promising alternatives for increasing CNTs water solubility and further neuron growth stimulation and cell membrane incorporation.

5.2. CNTs Application Strategies. The literature reports on two possible strategies to control neural cell functions through biofunctionalized CNTs. One is through the addition of soluble stand-alone CNTs directly to neuronal cell culture medium, while the other one involves CNTs surface modification for further attachment substrates such as scaffolds. The first strategy requires a direct application of the CNTs to the nerve tissue allowing the carbon structures to interact directly with nerve culture and to expand or disperse within the cells.

The second strategy employs CNTs as modifiers (either surface or bulk) of other materials to enhance their neurofunctionality as part of multicomposite scaffolds, implantable devices cell guiding matrices, and enhancing activity of other components such as the therapeutic drugs (small molecules), proteins (neurotrophic factors or extracellular matrix (ECM) components), and nucleic acids (siRNA, miRNA, pDNA, etc.) [43].

Properties like roughness, charge, polarity, and chemistry of CNT scaffolds, can alter the affinity of neurons linked to CNT-containing surfaces. Direct interactions between neurites and CNTs act as an exoskeleton, with more membrane/material tight junction formation. Greater surface area of CNT significantly results in stronger charge injection capacity and lesser interfacial impedance as they help in electrochemical coupling via electron transfer between CNTs and neurites [5, 44, 47, 48].

5.3. Neural Response Mechanisms to CNTs. CNTs have demonstrated to play an important role mediating interactions between neurons and their environment. When used as a scaffold, CNTs act not only as a reservoir of adsorbed proteins, but also play a dynamic role in boosting neuronal electrical performance. Observed discontinuous and tight contacts between MWCNT or SWNT bundles and neuronal membranes favor the hypothesis of a direct electrical coupling (Figure 5). The work of Cellot et al. demonstrate that meshwork of MWCNTs outside neurons is in intimate contact with a small area of the neuritic membranes, this 


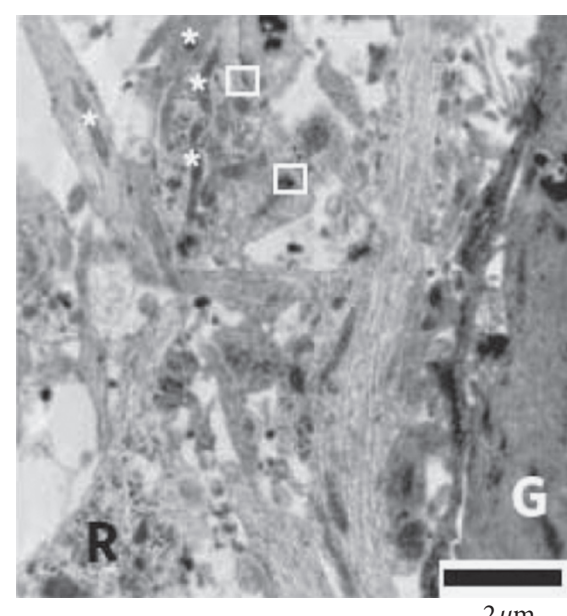

$2 \mu \mathrm{m}$

(a)

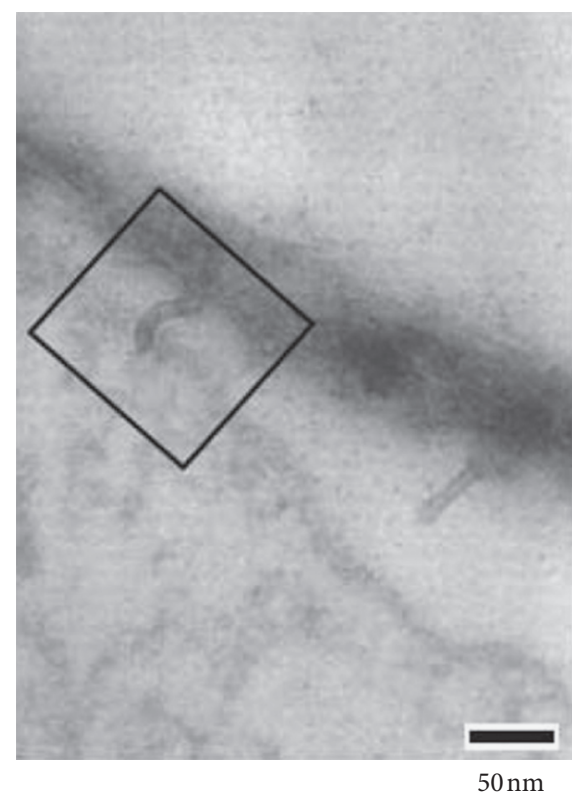

(c)

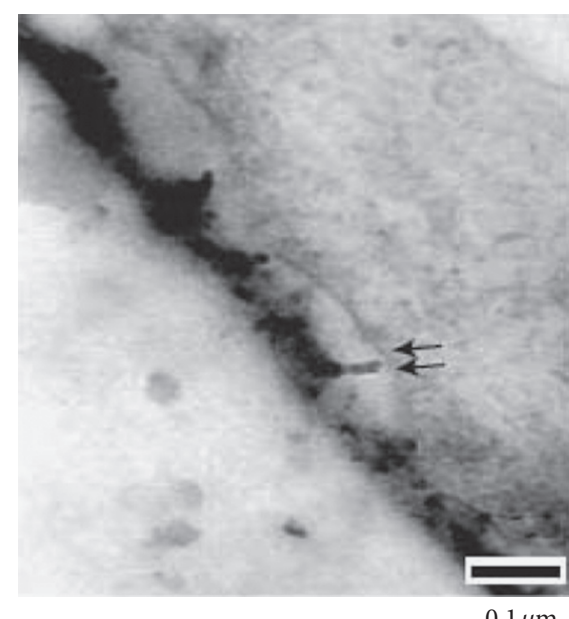

$0.1 \mu \mathrm{m}$

(b)

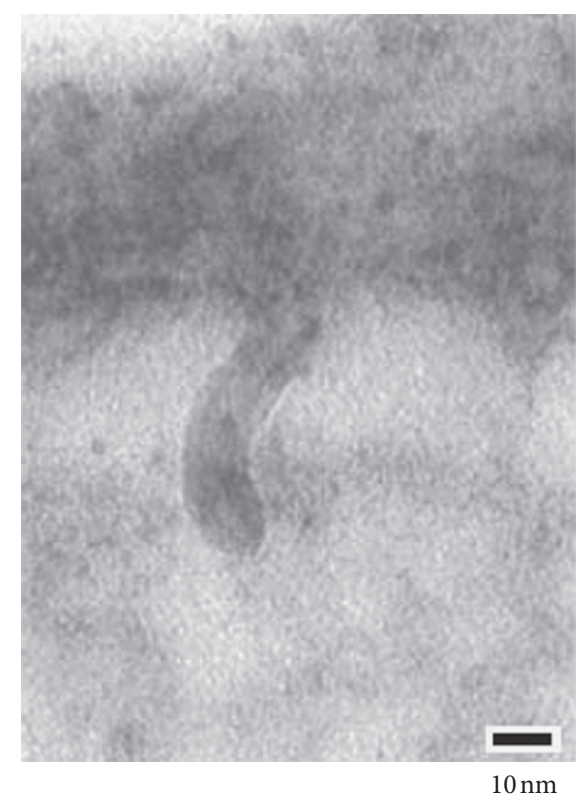

(d)

FIgURE 5: Interaction between MWCNTs and neurons. (a) Transmission electron micrographs (TEM) sections of neurons grown on MWCNTs showing functional synaptic contacts (rectangular box). (b) Arrows indicate MWCNT-membrane contacts on this TEM section from (a). (c, d) High-magnification micrographs from a section consecutive to those of (b) illustrating how MWCNTs 'pinch' neuronal membranes [10].

report constitutes the very first attempt at linking electrical phenomena in nanomaterials to neuron excitability [10].

Hippocampal neurons cultured on MWCNTs were studied by Fabbro et al. [49] demonstrating an increase in expression of paxillin, this membrane protein is involved in focal adhesions-mediated intracellular signaling pathways, demonstrating that electrophysiological cues provided by CNTs can be translated into specific neuronal signals. However, more detailed mechanistic studies between the neuronal tissue and CNTs interface are still required to engineer further applications of CBNs [5, 48, 50]. Some recent applications are presented in the following section.
5.4. Selected Applications. CNTs have been incorporated in the design and manufacturing of several biomedical technologies. A number of comprehensive reviews on the use of CNTs for neuron regeneration can be found in the literature $[7,15,22,51]$. This section aims to present recent examples of CNT-based materials used as scaffolds (hard printed or in the form of hydrogels) for neuron culture and conduits for nerve reconstruction. Most of the following subsections detail CNT-based materials applied to peripheral nerve studies; nevertheless, the first example is remarkable as it shows a rare example of CNT-mediated cortical regeneration. 
5.4.1. Transparent CNT-Based Substrates for Cortical Functional Regeneration. Pampaloni and coworkers have recently reported on the preparation of optically transparent CNT-based substrate (tCNTs) consisting of MWCNT carpets grown on fused silica substrates [52]. tCNTs not only favor dissociated primary neurons network formation and function (Figure 6(a)) but also boost the spontaneous synaptic activity of hippocampal neurons. In this report, tCNTs were used to support the growth of intact or lesioned entorhinal-hippocampal complex organotypic cultures (EHCs: 3D explants of the CNS in which the overall functional and anatomical neuronal connections are preserved [53]), finding that the CNT-based material induces the sprouting of functionally active fibers crossing lesioned areas (Figures 6(b) and 6(c)).

5.4.2. 3D Printing Nanoconductive MWCNT Scaffolds for Nerve Regeneration. Aminated MWCNTs have been recently been incorporated in a poly(ethylene glycol) diacrylate (PEGDA) matrix [54]. This report shows how CNTs can be easily incorporated in emerging $3 \mathrm{D}$ printing technologies to render scaffolds that support differentiation and growth of neural cells while having microelectroporous characteristics, and the manufacturing process is depicted in Figure 7.

In this study on MWCNT-PEGDA scaffolds, concentration of CNTs was evaluated, finding that proliferation at $0.02 \%$ of amine-containing MWCNT was the highest after four days of culture, whereas the highest proliferation of neural stem cells (NSCs) in all the concentration groups occurred in the $0.1 \%$ amine-containing MWCNT scaffolds. This proliferation was obtained until day seven, and the researchers believe that this delay was caused by the adaptation process of the NSCs to the substrate. Higher concentrations of MWCNTs in the printed scaffolds showed a higher positive charge which could promote a better development and greater nerve cell growth [54].

5.4.3. CNT-Interfaced Glass Fiber Scaffold for Regeneration of Transected Sciatic Nerve. Peripheral nerve injuries are common in clinical settings, yet the possibility of the nerve to regenerate spontaneously will vary according to the severity of the injury, which will be limited if the injury is too severe. The study by Ahn et al. addresses this issue by fabricating a phosphate glass microfiber (PGF) scaffold that incorporates aminated CNT [55].

The researchers performed a surface coupling of aminated CNTs to aligned PGF bundles and used the resulting fibers as interfacing material for neuron physical guidance. In vivo cell guidance studies were performed after wrapping the CNT-PGF substrate around poly(l/d-lactic acid) (PLDLA) electrospun nanofibers. The nerve guidance device was made in the shape of a cylindrical tube (as shown in Figure 8) and tested in a rat sciatic injury model [55], exhibiting good neural interaction, cell viability, and physicochemical integrity. Finally, the implant displays effectiveness in restoring motor functions, indicating that the muscle in the animal was functionally improved as a result of the CNT interfacing, as the scaffold-crossing axons reinnervated into the gastrocnemius muscles [55].

5.4.4. Polysaccharide/CNT Hydrogel Hybrid as Neuronal Growth Substrates. Hydrogels are attracting much attention in biomedical applications given their molecular-scale control over mechanical and bioresponsive properties [56]. Most hydrogels still lack good mechanical strength and electrical conductivity, thus limiting their biomedical applications, but CNT hydrogels hybrids have emerged as candidates to overcome this. These composite hydrogels have rapidly gained attention in developing regenerative therapies for skeletal muscles and cardiac and neural cells [57].

A recent study by $\mathrm{Wu}$ et al. explores the potential of chitin-based composite hydrogels incorporating MWCNTs [58]. These hybrid hydrogels originated from a chitin/ $\mathrm{NaOH} /$ urea aqueous solution blended with modified MWCNTs. Hydrogel bulk consisted on bundles of chitin nanofibers and carbon nanostructures that were stabilized through intermolecular forces like hydrogen bonding and electrostatic and hydrophobic interactions (Figure 9), that also contribute to refrain CNT release from the hydrogel scaffold. The resulting hydrogels showed improvements in thermal stability, a better hemocompatibility, good mechanical properties, while slowing down biodegradability rates, and the swelling ratio compared to control chitin hydrogels [58]. On top of these improvements, in vitro evaluation of Schwann cells was performed, resulting in a successful proliferation of the neuronal cells with little cytotoxicity and neurotoxicity, displaying a promising potential as neuronal growth substrates for peripheral nerve regeneration [58].

5.4.5. Nerve Guide Conduits Based on Protein/CNT Composites. The study presented by Mottaghitalab et al. introduced a clever nerve guide conduit (NGC) design that merged the mechanical advantages of the naturally occurring proteins silk fibroin (SF) and SWCNTs for use in nerve grafts. The resulting conduit showed stable chemical, physical, and electroconductivity properties due to its uniformity; plus the addition of fibronectin containing nanofibers (FN) through a electrospinning process rendered an addition extracellular matrix guidance for neuron growth and migration (Figure 10). FN conferred the SF/SWNTs NGCs conduits bioactivity allowing the growth and adhesion of U373 cell lines. NGCs were studied in vivo, implanted to $10 \mathrm{~mm}$ left sciatic nerve defects in rats, resulting in nerve regeneration in the proximal regions of the implants after five weeks. In both SF/SWNT and SF/SWNT/ FN NGCs, more myelinated axons were present as well as higher nerve conduction velocities, indicating a functional recovery for the injured nerves [59].

\section{Biocompatibility and Neurotoxicity of CNTs}

Academic communities and regulatory agencies have grown in concern about the adverse effects of CNT-based materials and nanomaterials in general. Factors such as particle concentration, exposure route (injection, ingestion, and 


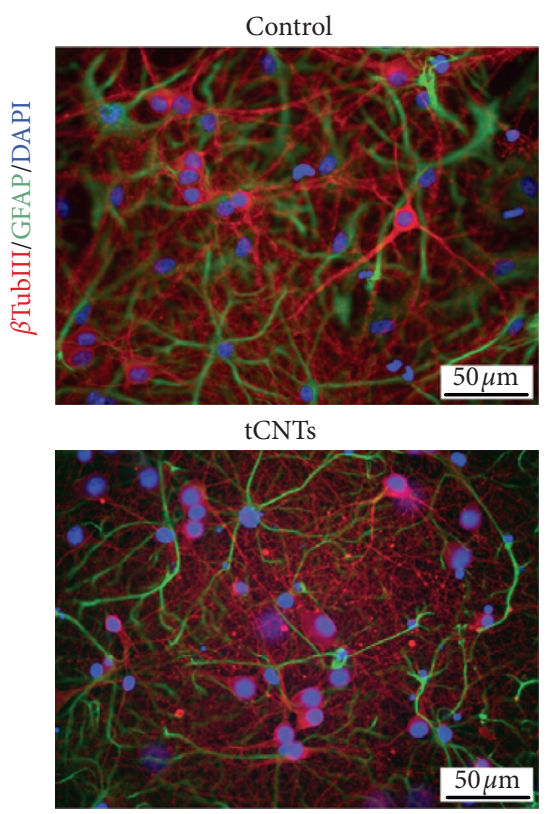

(a)
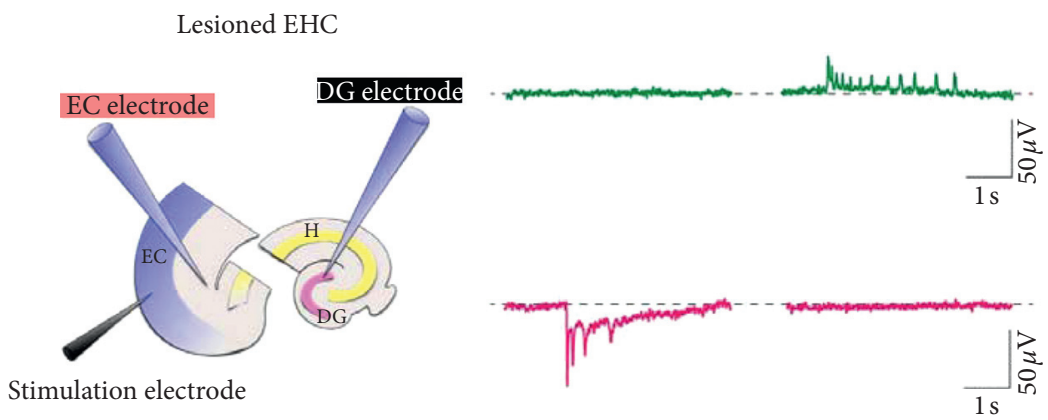

(b)
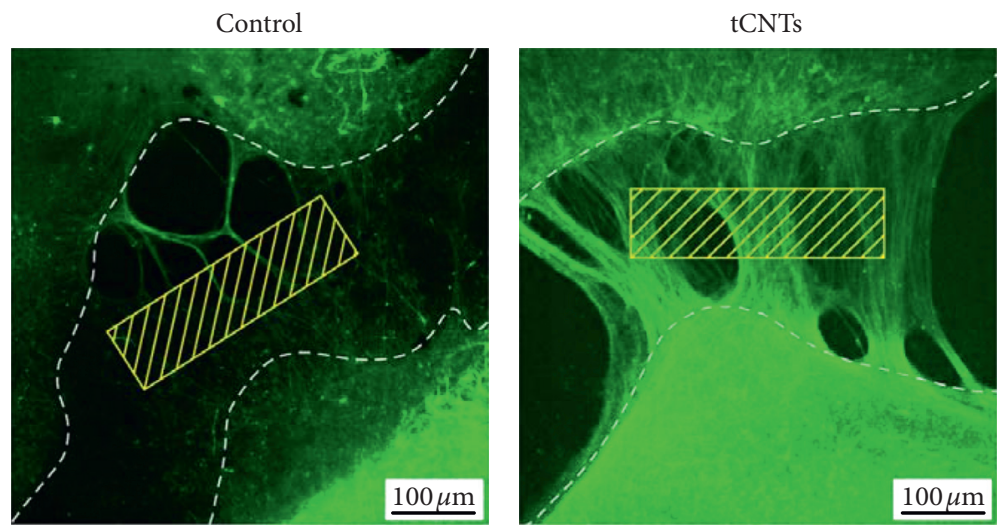

(c)

Figure 6: (a) Fluorescent micrographs of dissociated primary cells networks grown on glass control substrates (top) and on tCNTs substrates (bottom) ( $\beta$-tubulin III, red: neurons; GFAP, green: astrocytes; DAPI, blue: cell nuclei). (b) tCNTs induce the sprouting of functionally active fibers crossing lesioned areas in entorhinal-hippocampal complex organotypic cultures (EHCs). (a) Entorhinal cortex/ dentate gyrus (EC/DG) intercommunication ability through the perforant pathway (PP) transection in intact and lesioned EHC using a stimulation electrode inserted into the EC superficial layer. (c) Representative confocal images showing the sprouting of SMI32-positive axon fibers (in green) crossing the gap area [52].

inhalation [60]), particle size, particle distribution, particle agglomeration, and surface adsorbability and attachment are pivotal to comprehend the huge variability in the nanotoxicity studies of CNTs [61].

Most of these studies CNTs have been developed on stand-alone CNTs (i.e., suspended in physiological circulating fluids) generating accumulation-related adverse reactions in the tissues [62]. Nonetheless, when CNTs are firmly surface-immobilized (by either covalent or noncovalent approaches), it seems that there is no or there is little cytotoxicity [61]; that is the case of devices commonly inserted in the CNS/PNS directly in contact with neurons. Therefore, CNTs show in general good compatibility in vivo with neuronal tissues.
The most cautious way to use CNT would be to prevent them from entering the organism freely [60]. Examples like the one discussed in Section 5.4.4 or other CNThydrogel systems [63] comprise the trapping of free CNTs within the hydrogel network, thus limiting standalone CNTs release.

As long as CNTs remain firmly attached to a surface or their release is hampered, collateral diseases (such as cardiopulmonary diseases, inflammation and fibrosis, among others $[64,65])$ can be prevented. Then factors like superficial load, distribution of the functional groups of the CNTs, and manufacture methods $[65,66]$ and their relation to toxicological responses can be discussed, as we describe in the following section. 


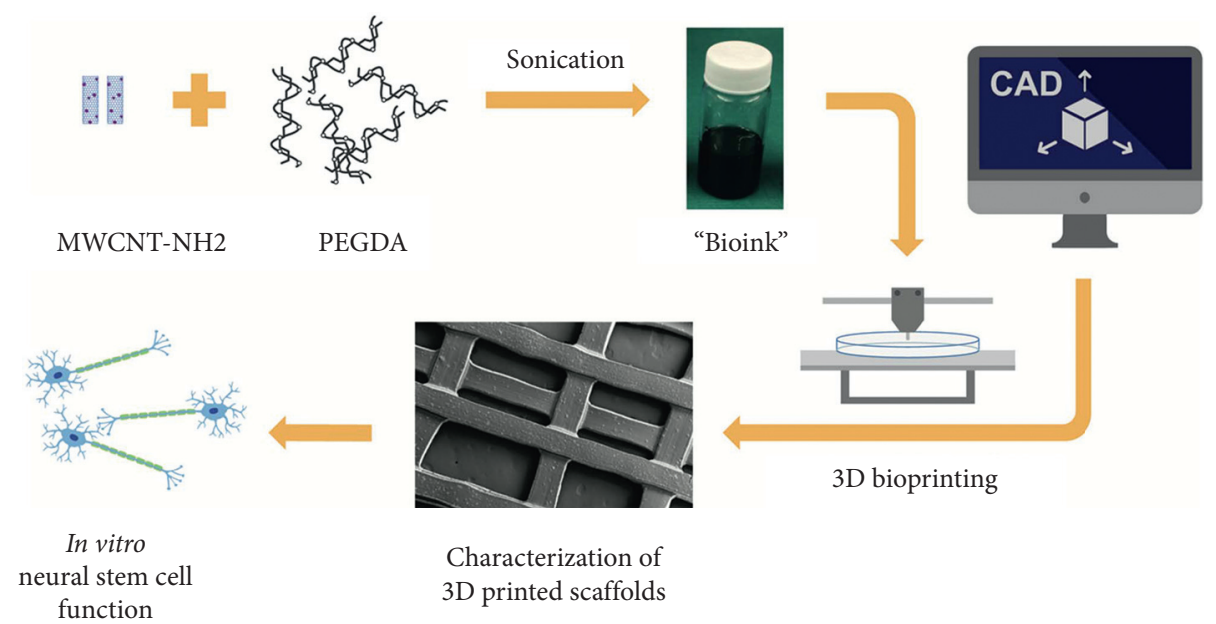

FIGURE 7: Representation of the manufacturing process of 3D-printing nanoconductive MWCNT-PEGDA scaffolds [54].
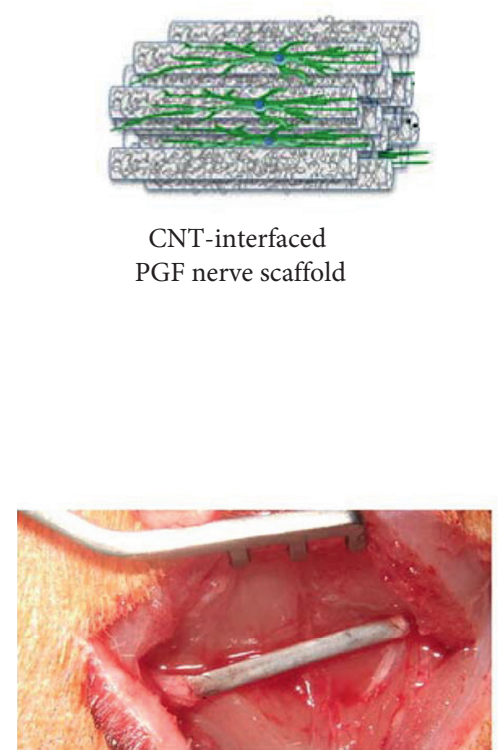

Implantation into transected sciatic nerve

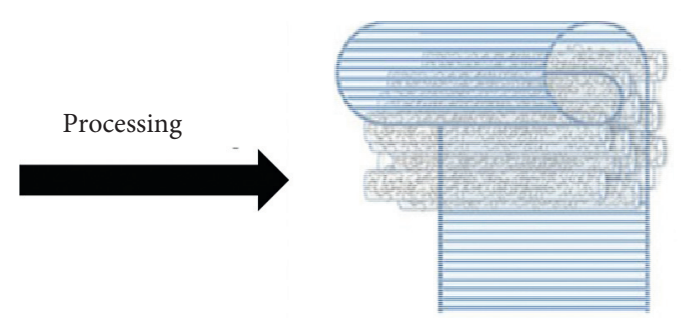

Wrapping with aligned nanofiber PLDLA
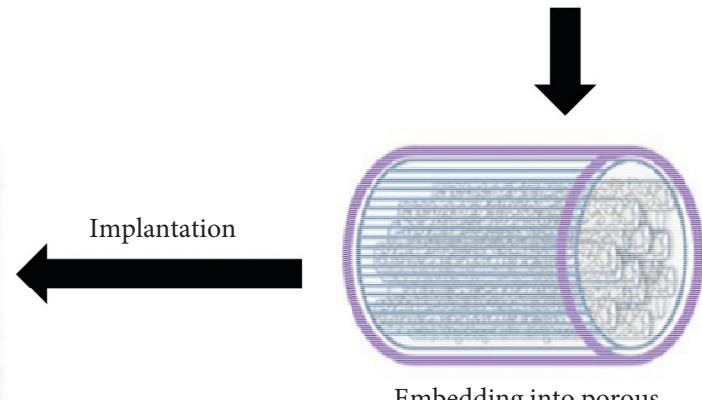

Embedding into porous PLDLA conduit

FIgURE 8: Functionality of a CNT-interfaced PGF scaffold used as nerve conduit. The aligned PGF bundle interfaced with CNTs for neurite outgrowth (adapted from reference [55]).

6.1. CNTs Neurotoxicity Related to Manufacturing and Functionalisation. As previously mentioned, CNT can be manufactured by different methods (arc discharge, chemical vapor deposition, and laser ablation of graphite among others) which generate a wide spectrum of CNT lengths, number of walls, chiralities, and most importantly impurities. Despite the fact that a number of studies highlight toxic effects in cells upon exposure to stand-alone CNTs, these adverse effects are largely due to heavy metal nanoparticles (Fe, Co, Ni, Y) produced during their synthesis [67].

Further functionalization steps tend to remove these metals and also to reduce CNTs' tendency to bundle, thus improving further biodistribution and lowering inflammatory responses compared to pristine CNTs [67-70]. Table 1 shows some toxicity-related effects as consequence of different CNTs solubilizing and functionalisation treatments in biological tissues with special emphasis on neuron related reports $[67,92]$.

On top of the chemical nature of impurities in CNTs, the number of walls, length, and chirality also influence their toxicity and behavior at biointerfaces [93, 94]. Depending on these physical characteristics, their cell interaction mechanism can be altered and generate different immunological responses [95, 96]. Several studies have shown a lower immune response as CNT length decreases, since short CNT can cross cell membranes more easily, whereas longer CNTs remain bundled in the 


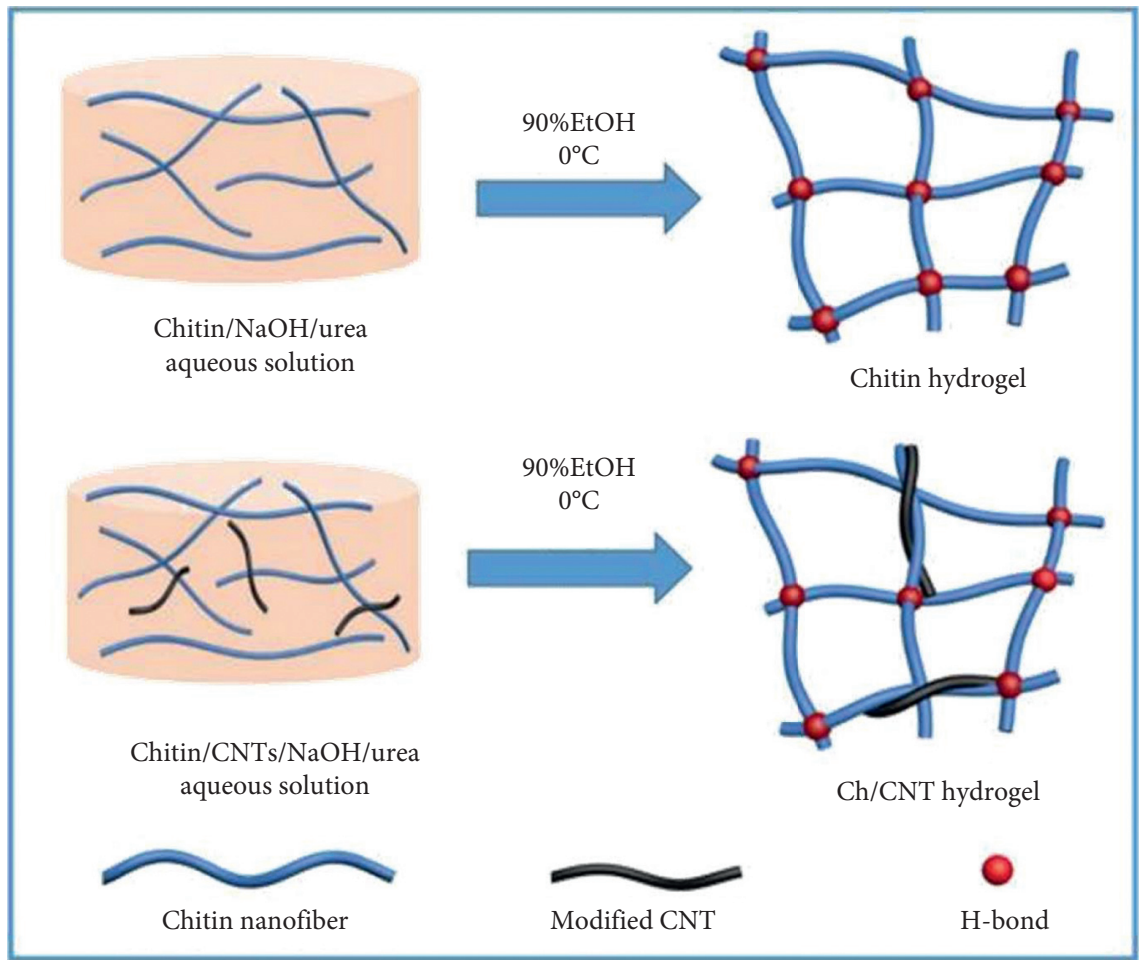

FIGURE 9: Schematic representation of the synthesis process of chitin-MWCNT hydrogel hybrids [58].
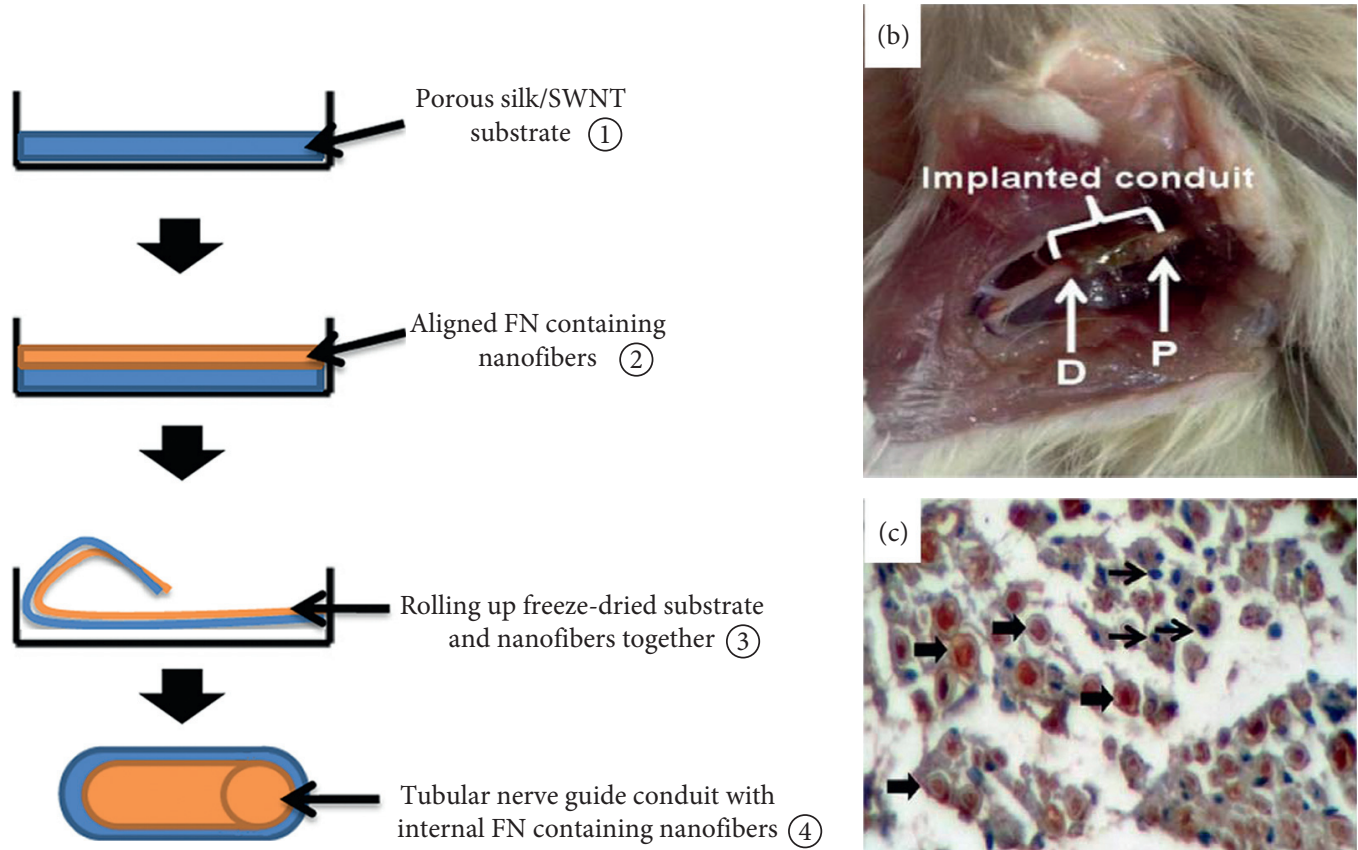

Rolling up freeze-dried substrate and nanofibers together (3)

Tubular nerve guide conduit with internal FN containing nanofibers (4)

(a)

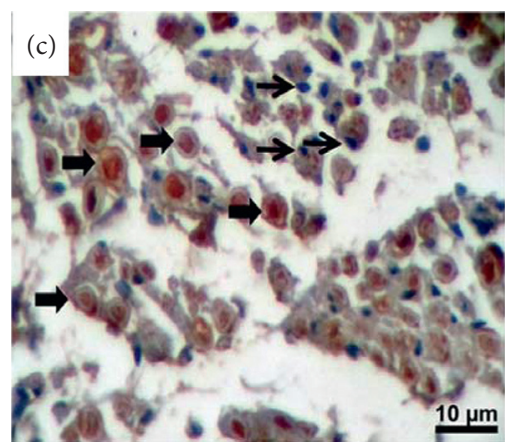

Figure 10: (a) Schematic representation of rolling up protocol for nerve guide conduit (NGC) containing SF/SWNT/FN. (1) Porous SF/ SWNT substrates. (2) Aligned FN containing nanofibers prepared by electrospinning on substrate. (3) Rolling up the complex. (4) Tubular NGC with internal aligned nanofibers coated by porous Silk/SWNT substrate. (b) Implanted SF/SWNT/FN. conduit in left sciatic nerve defects in a rat model after 5 weeks implantation. (c) Cross sections of regenerated nerves taken from SF/SWNT/FN nerve conduit after 5 weeks. Wide arrows: myelinated axon. Narrow arrows: Schwann cells (adapted from reference [59]). 
TABLE 1: Toxicity study of various carbon nanotubes solubilization and functionalisation schemes of biological interest.

\begin{tabular}{|c|c|c|}
\hline Small molecules as solubilizing agents & Toxicity & Reference \\
\hline Tetrahydrofuran & Tumorigen, mutagen & {$[71]$} \\
\hline Dichlorocarbene & Harmful & {$[71]$} \\
\hline Anthracene & Possible tumor promoter & {$[72]$} \\
\hline Pyrene & Carcinogenic, mutagenic & {$[73]$} \\
\hline Zn-porphyrin & Unknown, likely safe & {$[74]$} \\
\hline Phenylethyl alcohol & Topical irritant & {$[75]$} \\
\hline$n$-octyl- $\beta$-d-glucoside & Unknown & {$[76]$} \\
\hline$n$-decanoyl- $N$-methylglucamide & Unknown & {$[76]$} \\
\hline Triaminopyrimidine & Unknown & [77] \\
\hline Lysophosphatidylcholine & Unknown & {$[77]$} \\
\hline Barbituric acid & Not pharmacologically active & [78] \\
\hline Sodium cholate & Unknown & {$[76]$} \\
\hline Taurine & Safe up to $\sim 28.57 \mathrm{mg} / \mathrm{mL}$ & [79] \\
\hline Thiolated organosilane & Unknown & {$[80]$} \\
\hline Macromolecules as solubilizing agents & Toxicity & Reference \\
\hline Chitosan & Mostly safe & {$[81]$} \\
\hline Helical amylase & Unknown & {$[82]$} \\
\hline Poly(phenyleneethynylene) & Possible antimicrobial properties & [83] \\
\hline Poly(aminobenzene sulfonic acid) & Hazardous to blood, nervous system, liver & {$[84]$} \\
\hline PAA & Severely irritating and corrosive & [85] \\
\hline PEG & Acute oral and dermal & {$[84]$} \\
\hline Sulfonated polyaniline & Unknown & {$[86]$} \\
\hline Functionalisation approach & Toxicity & Reference \\
\hline MWCNT-NH${ }_{3}^{+}$ & Weak transient inflammatory response on glial cells & {$[87]$} \\
\hline${ }^{13} \mathrm{C}$ enriched SWCNTs + Tween-80 $1 \%$ & Moderate (mouse lungs and liver), biodistribution study & {$[88]$} \\
\hline PEG-modified SWCNTs & Mostly safe (spinal cord injury) & {$[89]$} \\
\hline${ }^{111}$ In]DTPA-MWCNTs & Not determined (blood-brain barrier in vitro model) & {$[90]$} \\
\hline PEG-oligodeoxynucleotide (CpG) & Mostly safe (glioma tumor model) & [91] \\
\hline
\end{tabular}

PEG: polyethylene glycol; PAA: poly(acrylic acid); DTPA: diethylenetriaminepentaacetic acid.

extracellular space [97] often generating reactive oxygen species (ROS), thus triggering oxidative stress response on cells [93].

6.2. Neuron Interaction Mechanisms. So far, it is understood that there are two possible mechanisms that CNTs can present to enter neurons or potential host cells: active transport through endocytosis/phagocytosis and passive transport or simple diffusion (also known as nanopenetration) [92].

Both mechanisms are presented in Figure 11, where the absorption of CNTs using the deformation of the plasma membrane to form a vesicle that internalizes into the cytoplasm is resented $[98,99]$. Phagocytosis is a process similar to endocytosis but is characterized by its specialized exogenous material of greater size as bacteria or microorganisms. Both mechanisms are dependent on energy (ATP) and temperature [100, 101].

Nanopenetration is a passive mechanism that allows the CNT to cross the membrane without the need to generate a vesicle, and it can be compared with the simple diffusion that some substances of interest for the membrane present [102]. The results of several studies of both mechanisms suggest that they can generate different immune reactions, as they activate a number of transport routes at the same time and also DNA damage can be involved at some point [102].
A number of studies have tested how a number of small and macromolecules can travel across the bloodbrain barrier (BBB) [103]; in fact, CNTs are no exception to this. When ${ }^{13} \mathrm{C}$-enriched SWCNTs were administered to mice, it was found they accumulate in the animal's brain, but showing little or no acute toxicity while also accumulating in liver, lung, and spleen, organs where CNTs persistence may lead to long-term toxicity effects [88].

MWCNTs are also able to cross the BBB as the work of Kafa et al. demonstrated. In this study, radiolabeled MWCNTs were intravenously administered to a murine model in order to study the molecular mechanism mediating CNTs crossing the $\mathrm{BBB}$, finding that micropinocytosis is the prevalent internalization mechanism, and therefore transcellular uptake is hypothesized as the primary mechanism behind the BBB crossing [90].

Gastrointestinal administration of SWCNTs can lead to accumulation across the BBB, it also known that SWCNTs tend to accumulate in neurons' lysosomes. Yang et al. took advantage of these observations to treat Alzheimer's disease model mice by delivering acetylcholine using the CNTs [104]. This study based the release of cargo based on a $\mathrm{pH}$ change in neuron lysosomes, but it has been demonstrated that CNTs can be enzymatically degraded by peroxidases in immune cells, glia cells, and the extracellular space as well [67]; therefore, lessening concerns about their use in neuron therapies. 


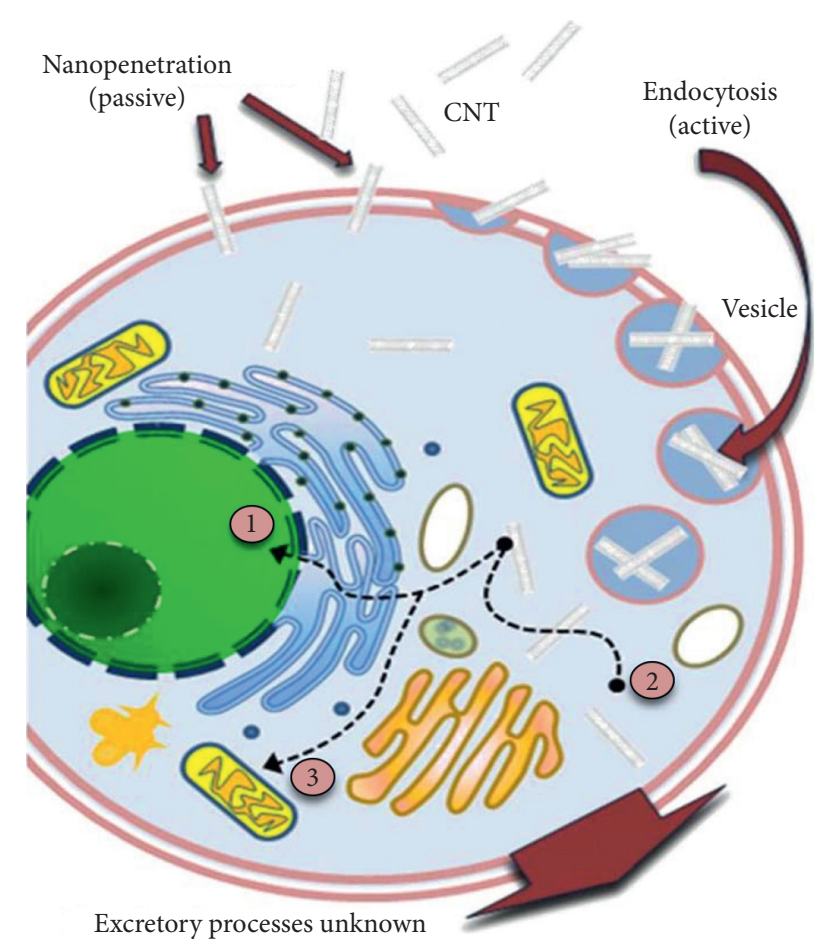

Potential targets and payload effects

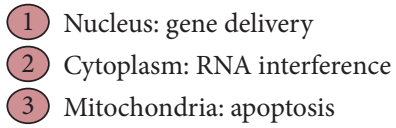

FIGURE 11: Schematics showing endocytosis and nanopenetration mechanisms of stand-alone CNTs [92].

\section{Concluding Remarks and Future Perspectives}

This review has examined how CBNs have been recently applied to nervous tissue regeneration. Research on nervous system repair continues to use CNTs in the pursue of novel therapies for treating nerve damage and neurodegenerative pathologies. It was found that most of the literature reports on neuronal applications of CNTs happen to take place on nervous cell lines or PNS; therefore, more research focused on CNS regeneration should be developed despite its complexity.

Even though initial safety concerns about stand-alone CNTs toxicity limited their use in neuronal applications, more and more studies show how these issues can be bypassed by controlling CNTs firm attachment to a substrate. Further nanotoxicological and pharmacokinetic studies are required, but it is highly advised for these reports to include extensive details of CNTs physical and chemical characterization of the $\mathrm{CBN}$; its impurities and surface characteristics in order to systematically homogenize the, quite contradictory, outcome results. In this regard, more studies should be carried out on the relation between toxicity and shape, size, functional groups, and release of CNT when integrated into devices.

As more control over CNT behavior is gained, the better comprehension of their interactions with neurons will be gained. This will facilitate a more efficient CNT incorporation a part of devices like nerve conduits, sensors, and micro and nanoelectromechanical systems (NEMS and MEMS). It is likely that future studies involving neuronal application of CNTs explore the benefits of combining different forms of CBNs, allowing for sensors and micro and nanoelectromechanical systems (NEMS and MEMS) with increased functionality and levels of complexity.

Current advances on additive manufacturing technologies like 3D printing will allow for increasing complexity of CNT based materials, this will provide improved compatibility and functionality of CNT-containing system when designed to match the intricacies of the CNS. Additive manufacturing technologies will also allow for the precise and reproducible merging of CNTs with biologically relevant cues like proteins and biodegradable biopolymers, thus improving the application of CNTs in the commission of nervous regeneration.

\section{Conflicts of Interest}

The authors declare no conflicts of interest.

\section{Authors' Contributions}

Conceptualization was done by J. V. Investigation was done by R. L., D. B., C. E., D. H., and C. R. Original draft was prepared by R. L., D. B., C. E., D. H., and C. R. Review and editing were performed by R. L., D. B., C. E., D. H., and C. R. C. R., J. V. supervised the study. J. V and C. R received the funding.

\section{Acknowledgments}

This research was funded by LANOTEC-CeNAT and the Ministry of Science Technology and Telecommunications of the Republic of Costa Rica (PINN-MICITT).

\section{References}

[1] A. Nunes, K. Al-Jamal, T. Nakajima, M. Hariz, and K. Kostarelos, "Application of carbon nanotubes in neurology: clinical perspectives and toxicological risks," Archives of Toxicology, vol. 86, no. 7, pp. 1009-1020, 2012.

[2] D. C. Rubinsztein, "The roles of intracellular protein-degradation pathways in neurodegeneration," Nature, vol. 443, no. 7113, pp. 780-786, 2006.

[3] A. Ajetunmobi, A. Prina-Mello, Y. Volkov, A. Corvin, and D. Tropea, "Nanotechnologies for the study of the central nervous system," Progress in Neurobiology, vol. 123, pp. 18-36, 2014.

[4] G. R. D. Evans, "Peripheral nerve injury: a review and approach to tissue engineered constructs," The Anatomical Record, vol. 263, no. 4, pp. 396-404, 2001.

[5] L. A. Pfister, M. Papaloïzos, H. P. Merkle, and B. Gander, "Nerve conduits and growth factor delivery in peripheral nerve repair," Journal of the Peripheral Nervous System, vol. 12, no. 2, pp. 65-82, 2007.

[6] J. T. Seil and T. J. Webster, "Electrically active nanomaterials as improved neural tissue regeneration scaffolds," Wiley Interdisciplinary Reviews: Nanomedicine and Nanobiotechnology, vol. 2, no. 6, pp. 635-647, 2010. 
[7] A. Fabbro, M. Prato, and L. Ballerini, "Carbon nanotubes in neuroregeneration and repair," Advanced Drug Delivery Reviews, vol. 65, no. 15, pp. 2034-2044, 2013.

[8] T. J. Webster, M. C. Waid, J. L. McKenzie, R. L. Price, and J. U. Ejiofor, "Nano-biotechnology: carbon nanofibres as improved neural and orthopaedic implants," Nanotechnology, vol. 15, no. 1, pp. 48-54, 2004.

[9] C. J. Serpell, K. Kostarelos, and B. G. Davis, "Can carbon nanotubes deliver on their promise in biology? Harnessing unique properties for unparalleled applications," ACS Central Science, vol. 2, no. 4, pp. 190-200, 2016.

[10] G. Cellot, E. Cilia, S. Cipollone et al., "Carbon nanotubes might improve neuronal performance by favouring electrical shortcuts," Nature Nanotechnology, vol. 4, no. 2, pp. 126-133, 2009.

[11] C. C. Liu, J. J. Zhao, R. Zhang et al., "Multifunctionalization of graphene and graphene oxide for controlled release and targeted delivery of anticancer drugs," American Journal of Translational Research, vol. 9, no. 9, pp. 5197-5219, 2017.

[12] A. Fabbro, S. Bosi, L. Ballerini, and M. Prato, "Carbon nanotubes: artificial nanomaterials to engineer single neurons and neuronal networks," ACS Chemical Neuroscience, vol. 3, no. 8, pp. 611-618, 2012.

[13] J. S. Belkas, M. S. Shoichet, and R. Midha, "Peripheral nerve regeneration through guidance tubes," Neurological Research, vol. 26, no. 2, pp. 151-160, 2004.

[14] N. Zhang, H. Yan, and X. Wen, "Tissue-engineering approaches for axonal guidance," Brain Research Reviews, vol. 49, no. 1, pp. 48-64, 2005.

[15] A. Fraczek-Szczypta, "Carbon nanomaterials for nerve tissue stimulation and regeneration," Materials Science and Engineering: $C$, vol. 34, pp. 35-49, 2014.

[16] J. S. H. Taylor and E. T. W. Bampton, "Factors secreted by schwann cells stimulate the regeneration of neonatal retinal ganglion cells," Journal of Anatomy, vol. 204, no. 1, pp. 25-31, 2004.

[17] C. Zhao, A. Tan, G. Pastorin, and H. K. Ho, "Nanomaterial scaffolds for stem cell proliferation and differentiation in tissue engineering," Biotechnology Advances, vol. 31, no. 5, pp. 654-668, 2013.

[18] K. Liu, A. Tedeschi, K. K. Park, and Z. He, "Neuronal intrinsic mechanisms of axon regeneration," Annual Review of Neuroscience, vol. 34, no. 1, pp. 131-152, 2011.

[19] E. Biazar, "Types of neural guides and using nanotechnology for peripheral nerve reconstruction," International Journal of Nanomedicine, vol. 839, 2010.

[20] M. Perán, M. García, E. Lopez-Ruiz, G. Jiménez, and J. Marchal, "How can nanotechnology help to repair the body? Advances in cardiac, skin, bone, cartilage and nerve tissue regeneration," Materials, vol. 6, no. 4, pp. 1333-1359, 2013.

[21] Z. Zhou, X. Liu, W. Wu et al., "Effective nerve cell modulation by electrical stimulation of carbon nanotube embedded conductive polymeric scaffolds," Biomaterials Science, vol. 6, no. 9, pp. 2375-2385, 2018.

[22] K. M. Oprych, R. L. D. Whitby, S. V. Mikhalovsky, P. Tomlins, and J. Adu, "Repairing peripheral nerves: is there a role for carbon nanotubes?," Advanced Healthcare Materials, vol. 5, no. 11, pp. 1253-1271, 2016.

[23] G. Jia, H. Wang, L. Yan et al., "Cytotoxicity of carbon nanomaterials: single-wall nanotube, multi-wall nanotube, and fullerene," Environmental Science \& Technology, vol. 39, no. 5, pp. 1378-1383, 2005.
[24] R. A. MacDonald, C. M. Voge, M. Kariolis, and J. P. Stegemann, "Carbon nanotubes increase the electrical conductivity of fibroblast-seeded collagen hydrogels," Acta Biomaterialia, vol. 4, no. 6, pp. 1583-1592, 2008.

[25] Z. Zhu, L. Garcia-Gancedo, A. J. Flewitt, H. Xie, F. Moussy, and W. I. Milne, "A critical review of glucose biosensors based on carbon nanomaterials: carbon nanotubes and graphene," Sensors, vol. 12, no. 5, pp. 5996-6022, 2012.

[26] C. Cha, S. R. Shin, N. Annabi, M. R. Dokmeci, and A. Khademhosseini, "Carbon-based nanomaterials: multifunctional materials for biomedical engineering," ACS Nano, vol. 7, no. 4, pp. 2891-2897, 2013.

[27] N. Choudhary, S. Hwang, and W. Choi, "Carbon nanomaterials: a review," in Handbook of Nanomaterials Properties, pp. 709-769, Springer, Berlin, Germany, 2014.

[28] R. F. Schinazi, R. Sijbesma, G. Srdanov, C. L. Hill, and F. Wudl, "Synthesis and virucidal activity of a water-soluble, configurationally stable, derivatized C60 fullerene," Antimicrobial Agents and Chemotherapy, vol. 37, no. 8, pp. 1707-1710, 1993.

[29] S.-R. Chae, Y. Watanabe, and M. R. Wiesner, "Comparative photochemical reactivity of spherical and tubular fullerene nanoparticles in water under ultraviolet (UV) irradiation," Water Research, vol. 45, no. 1, pp. 308-314, 2011.

[30] A. Husen and K. Siddiqi, "Carbon and fullerene nanomaterials in plant system," Journal of Nanobiotechnology, vol. 12, no. 1, p. 16, 2014.

[31] M. Srikanth, R. Asmatulu, K. Cluff, and L. Yao, "Material characterization and bioanalysis of hybrid scaffolds of carbon nanomaterial and polymer nanofibers," ACS Omega, vol. 4, no. 3, pp. 5044-5051, 2019.

[32] A. Nasri, A. Boubaker, B. Hafsi, W. Khaldi, and A. Kalboussi, "High-sensitivity sensor using C60-single molecule transistor," IEEE Sensors Journal, vol. 18, no. 1, pp. 248-254, 2018.

[33] S. He and W. Chen, "3D graphene nanomaterials for binderfree supercapacitors: scientific design for enhanced performance," Nanoscale, vol. 7, no. 16, pp. 6957-6990, 2015.

[34] P. Kumar, P. Huo, R. Zhang, and B. Liu, "Antibacterial properties of graphene-based nanomaterials," Nanomaterials, vol. 9, no. 5, p. 737, 2019.

[35] N. P. Pampaloni, M. Lottner, M. Giugliano et al., "Singlelayer graphene modulates neuronal communication and augments membrane ion currents," Nature Nanotechnology, vol. 13, no. 8, pp. 755-764, 2018.

[36] M. Pumera, "Graphene-based nanomaterials and their electrochemistry," Chemical Society Reviews, vol. 39, no. 11, p. 4146, 2010.

[37] A. I. Rusanov, "Thermodynamics of graphene," Surface Science Reports, vol. 69, no. 4, pp. 296-324, 2014.

[38] P.-C. Ma, N. A. Siddiqui, G. Marom, and J.-K. Kim, "Dispersion and functionalization of carbon nanotubes for polymer-based nanocomposites: a review," Composites Part A: Applied Science and Manufacturing, vol. 41, no. 10, pp. 1345-1367, 2010.

[39] R. Vidu, M. Rahman, M. Mahmoudi, M. Enachescu, T. D. Poteca, and I. Opris, "Nanostructures: a platform for brain repair and augmentation," Frontiers in Systems Neuroscience, vol. 8, p. 91, 2014.

[40] P. Gupta and D. Lahiri, "Aligned carbon nanotube containing scaffolds for neural tissue regeneration," Neural Regeneration Research, vol. 11, no. 7, pp. 1062-1603, 2016.

[41] C. Gao, Z. Guo, J.-H. Liu, and X.-J. Huang, "The new age of carbon nanotubes: an updated review of functionalized 
carbon nanotubes in electrochemical sensors," Nanoscale, vol. 4, no. 6, pp. 1948-1963, 2012.

[42] Y. Zhou, Y. Fang, and R. Ramasamy, "Non-covalent functionalization of carbon nanotubes for electrochemical biosensor development," Sensors, vol. 19, no. 2, p. 392, 2019.

[43] J.-Y. Hwang, U. S. Shin, W.-C. Jang, J. K. Hyun, I. B. Wall, and H.-W. Kim, "Biofunctionalized carbon nanotubes in neural regeneration: a mini-review," Nanoscale, vol. 5, no. 2, pp. 487-497, 2013.

[44] K. Matsumoto, C. Sato, Y. Naka, R. Whitby, and N. Shimizu, "Stimulation of neuronal neurite outgrowth using functionalized carbon nanotubes," Nanotechnology, vol. 21, no. 11, Article ID 115101, 2010.

[45] A. Sucapane, G. Cellot, M. Prato, M. Giugliano, V. Parpura, and L. Ballerini, "Interactions between cultured neurons and carbon nanotubes: a nanoneuroscience vignette," Journal of Nanoneuroscience, vol. 1, no. 1, pp. 10-16, 2009.

[46] C. Redondo-Gómez, F. Orozco, P.-L. Michael Noeske, V. Soto-Tellini, Y. R. Corrales-Ureña, and J. Vega-Baudrit, "Cholic acid covalently bound to multi-walled carbon nanotubes: improvements on dispersion stability," Materials Chemistry and Physics, vol. 200, pp. 331-341, 2017.

[47] V. Lovat, D. Pantarotto, L. Lagostena et al., "Carbon nanotube substrates boost neuronal electrical signaling," Nano Letters, vol. 5, no. 6, pp. 1107-1110, 2005.

[48] N. W. S. Kam, E. Jan, and N. A. Kotov, "Electrical stimulation of neural stem cells mediated by humanized carbon nanotube composite made with extracellular matrix protein," Nano Letters, vol. 9, no. 1, pp. 273-278, 2009.

[49] A. Fabbro, A. Sucapane, F. M. Toma et al., "Adhesion to carbon nanotube conductive scaffolds forces action-potential appearance in immature rat spinal neurons," PLoS One, vol. 8, no. 8, Article ID e73621, 2013.

[50] P. A. Tran, L. Zhang, and T. J. Webster, "Carbon nanofibers and carbon nanotubes in regenerative medicine," Advanced Drug Delivery Reviews, vol. 61, no. 12, pp. 1097-1114, 2009.

[51] Y. Zhang, Y. Bai, and B. Yan, "Functionalized carbon nanotubes for potential medicinal applications," Drug Discovery Today, vol. 15, no. 11-12, pp. 428-435, 2010.

[52] N. P. Pampaloni, I. Rago, I. Calaresu et al., "Transparent carbon nanotubes promote the outgrowth of enthorinodentate projections in lesioned organ slice cultures," Developmental Neurobiology, pp. 1-16, 2019.

[53] A. Vlachos, D. Becker, P. Jedlicka, R. Winkels, J. Roeper, and T. Deller, "Entorhinal denervation induces homeostatic synaptic scaling of excitatory postsynapses of dentate granule cells in mouse organotypic slice cultures," PLoS One, vol. 7, no. 3, Article ID e32883, 2012.

[54] S.-J. Lee, W. Zhu, M. Nowicki et al., “3D printing nano conductive multi-walled carbon nanotube scaffolds for nerve regeneration," Journal of Neural Engineering, vol. 15, no. 1, Article ID 016018, 2018.

[55] H.-S. Ahn, J.-Y. Hwang, M. S. Kim et al., "Carbon-nanotubeinterfaced glass fiber scaffold for regeneration of transected sciatic nerve," Acta Biomaterialia, vol. 13, pp. 324-334, 2015.

[56] Y. S. Zhang and A. Khademhosseini, "Advances in engineering hydrogels,” Science, vol. 356, no. 6337, 2017.

[57] A. Vashist, A. Kaushik, A. Vashist et al., "Advances in carbon nanotubes-hydrogel hybrids in nanomedicine for therapeutics," Advanced Healthcare Materials, vol. 7, no. 9, Article ID 1701213, 2018.

[58] S. Wu, B. Duan, A. Lu, Y. Wang, Q. Ye, and L. Zhang, "Biocompatible chitin/carbon nanotubes composite hydrogels as neuronal growth substrates," Carbohydrate Polymers, vol. 174, pp. 830-840, 2017.

[59] F. Mottaghitalab, M. Farokhi, A. Zaminy et al., "A biosynthetic nerve guide conduit based on silk/SWNT/fibronectin nanocomposite for peripheral nerve regeneration," PLoS One, vol. 8, no. 9, Article ID e74417, 2013.

[60] J. Simon, E. Flahaut, and M. Golzio, "Overview of carbon nanotubes for biomedical applications," Materials, vol. 12, no. 4, p. 624, 2019.

[61] L. Yan, F. Zhao, S. Li, Z. Hu, and Y. Zhao, "Low-toxic and safe nanomaterials by surface-chemical design, carbon nanotubes, fullerenes, metallofullerenes, and graphenes," Nanoscale, vol. 3, no. 2, pp. 362-382, 2011.

[62] C.-W. Lam, J. T. James, R. McCluskey, S. Arepalli, and R. L. Hunter, "A review of carbon nanotube toxicity and assessment of potential occupational and environmental health risks," Critical Reviews in Toxicology, vol. 36, no. 3, pp. 189-217, 2006.

[63] R. Bellingeri, F. Alustiza, N. Picco et al., "In vitro toxicity evaluation of hydrogel-carbon nanotubes composites on intestinal cells," Journal of Applied Polymer Science, vol. 132, no. 5, p. 41370, 2015.

[64] X. Chen, J. Fang, Y. Cheng et al., "Biomolecular interaction analysis for carbon nanotubes and for biocompatibility prediction," Analytical Biochemistry, vol. 505, pp. 1-7, 2016.

[65] N. M. Bardhan, D. Ghosh, and A. M. Belcher, "Carbon nanotubes as in vivo bacterial probes," Nature Communications, vol. 5, no. 1, p. 4918, 2014.

[66] C. Ge, J. Du, L. Zhao et al., "Binding of blood proteins to carbon nanotubes reduces cytotoxicity," Proceedings of the National Academy of Sciences, vol. 108, no. 41, pp. 16968 16973, 2011.

[67] M. Baldrighi, M. Trusel, R. Tonini, and S. Giordani, "Carbon nanomaterials interfacing with neurons: an in vivo perspective," Frontiers in Neuroscience, vol. 10, 2016.

[68] R. Singh, D. Pantarotto, D. McCarthy et al., "Binding and condensation of plasmid DNA onto functionalized carbon nanotubes: toward the construction of nanotube-based gene delivery vectors," Journal of the American Chemical Society, vol. 127 , no. 12, pp. 4388-4396, 2005.

[69] R. Singh, D. Pantarotto, L. Lacerda et al., "Tissue biodistribution and blood clearance rates of intravenously administered carbon nanotube radiotracers," Proceedings of the National Academy of Sciences, vol. 103, no. 9, pp. 3357-3362, 2006.

[70] G. Pagona and N. Tagmatarchis, "Carbon nanotubes: materials for medicinal chemistry and biotechnological applications," Current Medicinal Chemistry, vol. 13, no. 15, pp. 1789-1798, 2006

[71] H. Hu, B. Zhao, M. A. Hamon, K. Kamaras, M. E. Itkis, and R. C. Haddon, "Sidewall functionalization of single-walled carbon nanotubes by addition of dichlorocarbene," Journal of the American Chemical Society, vol. 125, no. 48, pp. 14893-14900, 2003.

[72] T. G. Hedderman, S. M. Keogh, G. Chambers, and H. J. Byrne, "In-depth study into the interaction of single walled carbon nanotubes with anthracene and p -terphenyl," The Journal of Physical Chemistry B, vol. 110, no. 9, pp. 3895-3901, 2006.

[73] D. M. Guldi, G. M. A. Rahman, N. Jux et al., "Functional single-wall carbon nanotube nanohybrids associating SWNTs with water-soluble enzyme model systems," Journal of the American Chemical Society, vol. 127, no. 27, pp. 9830-9838, 2005. 
[74] F. Cheng and A. Adronov, "Noncovalent functionalization and solubilization of carbon nanotubes by using a conjugated Zn-porphyrin polymer," Chemistry-A European Journal, vol. 12, no. 19, pp. 5053-5059, 2006.

[75] S. Dumonteil, A. Demortier, S. Detriche et al., "Dispersion of carbon nanotubes using organic solvents," Journal of Nanoscience and Nanotechnology, vol. 6, no. 5, pp. 13151318, 2006.

[76] A. Ishibashi and N. Nakashima, "Individual dissolution of single-walled carbon nanotubes in aqueous solutions of steroid or sugar compounds and their Raman and near-IR spectral properties," Chemistry-A European Journal, vol. 12, no. 29, pp. 7595-7602, 2006.

[77] A. P. Roberts, A. S. Mount, B. Seda et al., "In vivo biomodification of lipid-coated carbon nanotubes by Daphnia magna," Environmental Science \& Technology, vol. 41, no. 8, pp. 3025-3029, 2007.

[78] A. Ikeda, Y. Tanaka, K. Nobusawa, and J.-I. Kikuchi, "Solubilization of single-walled carbon nanotubes by supramolecular complexes of barbituric acid and triaminopyrimidines," Langmuir, vol. 23, no. 22, pp. 10913-10915, 2007.

[79] X. Wang, X. Y. Deng, H. F. Wang et al., "Bio-effects of water soluble taurine multi-wall carbon nanotubes on lungs of mice," Zhonghua Yu Fang Yi Xue Za Zhi, vol. 41, pp. 85-90, 2007.

[80] M. Bottini, A. Magrini, N. Rosato, A. Bergamaschi, and T. Mustelin, "Dispersion of pristine single-walled carbon nanotubes in water by a thiolated organosilane: application in supramolecular nanoassemblies," The Journal of Physical Chemistry B, vol. 110, no. 28, pp. 13685-13688, 2006.

[81] J. Tkac, J. W. Whittaker, and T. Ruzgas, "The use of single walled carbon nanotubes dispersed in a chitosan matrix for preparation of a galactose biosensor," Biosensors and Bioelectronics, vol. 22, no. 8, pp. 1820-1824, 2007.

[82] O.-K. Kim, J. Je, J. W. Baldwin, S. Kooi, P. E. Pehrsson, and L. J. Buckley, "Solubilization of single-wall carbon nanotubes by supramolecular encapsulation of helical amylose," Journal of the American Chemical Society, vol. 125, no. 15, pp. 4426-4427, 2003.

[83] J. Mao, Q. Liu, X. Lv et al., “A water-soluble hybrid material of single-walled carbon nanotubes with an amphiphilic poly(phenyleneethynylene): preparation, characterization, and photovoltaic properties," Journal of Nanoscience and Nanotechnology, vol. 7, no. 8, pp. 2709-2718, 2007.

[84] B. Zhao, H. Hu, A. Yu, D. Perea, and R. C. Haddon, "Synthesis and characterization of water soluble singlewalled carbon nanotube graft copolymers," Journal of the American Chemical Society, vol. 127, no. 22, pp. 8197-8203, 2005.

[85] A. Liu, T. Watanabe, I. Honma, J. Wang, and H. Zhou, "Effect of solution $\mathrm{pH}$ and ionic strength on the stability of poly(acrylic acid)-encapsulated multiwalled carbon nanotubes aqueous dispersion and its application for $\mathrm{NADH}$ sensor," Biosensors and Bioelectronics, vol. 22, no. 5, pp. 694-699, 2006.

[86] H. Zhang, H. X. Li, and H. M. Cheng, "Water-soluble multiwalled carbon nanotubes functionalized with sulfonated polyaniline," The Journal of Physical Chemistry B, vol. 110, no. 18, pp. 9095-9099, 2006.

[87] G. Bardi, A. Nunes, L. Gherardini et al., "Functionalized carbon nanotubes in the brain: cellular internalization and neuroinflammatory responses," PLoS One, vol. 8, no. 11, Article ID e80964, 2013.
[88] S.-T. Yang, W. Guo, Y. Lin et al., "Biodistribution of pristine single-walled carbon nanotubes in vivo $\dagger$," The Journal of Physical Chemistry C, vol. 111, no. 48, pp. 17761-17764, 2007.

[89] J. A. Roman, T. L. Niedzielko, R. C. Haddon, V. Parpura, and C. L. Floyd, "Single-walled carbon nanotubes chemically functionalized with polyethylene glycol promote tissue repair in a rat model of spinal cord injury," Journal of Neurotrauma, vol. 28, no. 11, pp. 2349-2362, 2011.

[90] H. Kafa, J. T.-W. Wang, N. Rubio et al., “The interaction of carbon nanotubes with an in vitro blood-brain barrier model and mouse brain in vivo," Biomaterials, vol. 53, pp. 437-452, 2015.

[91] D. Zhao, D. Alizadeh, L. Zhang et al., "Carbon nanotubes enhance CpG uptake and potentiate antiglioma immunity," Clinical Cancer Research, vol. 17, no. 4, pp. 771-782, 2011.

[92] C. P. Firme and P. R. Bandaru, "Toxicity issues in the application of carbon nanotubes to biological systems," Nanomedicine: Nanotechnology, Biology and Medicine, vol. 6, no. 2, pp. 245-256, 2010.

[93] D. Mohanta, S. Patnaik, S. Sood, and N. Das, "Carbon nanotubes: evaluation of toxicity at biointerfaces," Journal of Pharmaceutical Analysis, vol. 9, no. 5, pp. 293-300, 2019.

[94] R. Alshehri, A. M. Ilyas, A. Hasan, A. Arnaout, F. Ahmed, and A. Memic, "Carbon nanotubes in biomedical applications: factors, mechanisms, and remedies of toxicity," Journal of Medicinal Chemistry, vol. 59, no. 18, pp. 8149-8167, 2016.

[95] K. Kostarelos, "Rational design and engineering of delivery systems for therapeutics: biomedical exercises in colloid and surface science," Advances in Colloid and Interface Science, vol. 106, no. 1-3, pp. 147-168, 2003.

[96] A. Nel, "Toxic potential of materials at the nanolevel," Science, vol. 311, no. 5761, pp. 622-627, 2006.

[97] Y. Sato, A. Yokoyama, K.-I. Shibata et al., "Influence of length on cytotoxicity of multi-walled carbon nanotubes against human acute monocytic leukemia cell line THP-1 in vitro and subcutaneous tissue of rats in vivo," Molecular BioSystems, vol. 1, no. 2, p. 176, 2005.

[98] M. Marsh and H. T. McMahon, "The structural era of endocytosis," Science, vol. 285, no. 5425, pp. 215-220, 1999.

[99] G. J. Doherty and H. T. McMahon, "Mechanisms of endocytosis," Annual Review of Biochemistry, vol. 78, no. 1, pp. 857-902, 2009.

[100] N. W. S. Kam, Z. Liu, and H. Dai, "Carbon nanotubes as intracellular transporters for proteins and DNA: an investigation of the uptake mechanism and pathway," Angewandte Chemie International Edition, vol. 45, no. 4, pp. 577-581, 2006.

[101] N. W. Shi Kam, T. C. Jessop, P. A. Wender, and H. Dai, "Nanotube molecular transporters: internalization of carbon nanotube-protein conjugates into mammalian cells," Journal of the American Chemical Society, vol. 126, no. 22, pp. 6850-6851, 2004.

[102] M. Pacurari, X. J. Yin, J. Zhao et al., "Raw single-wall carbon nanotubes induce oxidative stress and activate MAPKs, AP$1, \mathrm{NF}-\kappa \mathrm{B}$, and akt in normal and malignant human mesothelial cells," Environmental Health Perspectives, vol. 116, no. 9, pp. 1211-1217, 2008.

[103] R. Daneman and A. Prat, "The blood-brain barrier," Cold Spring Harbor Perspectives in Biology, vol. 7, no. 1, 2015.

[104] Z. Yang, Y. Zhang, Y. Yang et al., "Pharmacological and toxicological target organelles and safe use of single-walled carbon nanotubes as drug carriers in treating Alzheimer disease," Nanomedicine: Nanotechnology, Biology and Medicine, vol. 6, no. 3, pp. 427-441, 2010. 